\title{
BMJ Open Liver dysfunction in idiopathic pulmonary arterial hypertension: prevalence, characteristics and prognostic significance, a retrospective cohort study in China
}

Cijun Luo (1) , ${ }^{1}$ Wenhui Wu, ${ }^{1}$ Changwei Wu, ${ }^{2}$ Hongling Qiu, ${ }^{1}$ Ping Yuan, ${ }^{1}$ Rong Jiang (1) , ${ }^{1}$ Qinhua Zhao, ${ }^{1}$ Sugang Gong, ${ }^{3}$ Rui Zhang, ${ }^{1}$ Jinling $\mathrm{Li}^{,}{ }^{1}$ Jing He, ${ }^{1}$ Jinming Liu, ${ }^{1}$ Lan Wang ${ }^{1}$

To cite: Luo C, Wu W, Wu C, et al. Liver dysfunction in idiopathic pulmonary arterial hypertension: prevalence, characteristics and prognostic significance, a retrospective cohort study in China. BMJ Open 2021;11:e045165. doi:10.1136/ bmjopen-2020-045165

- Prepublication history and additional supplemental material for this paper are available online. To view these files, please visit the journal online (http://dx.doi.org/10.1136/ bmjopen-2020-045165).

$\mathrm{CL}, \mathrm{WW}, \mathrm{CW}$ and $\mathrm{HQ}$ contributed equally.

Received 28 September 2020 Accepted 25 August 2021

Check for updates

(C) Author(s) (or their employer(s)) 2021. Re-use permitted under CC BY-NC. No commercial re-use. See rights and permissions. Published by BMJ.

For numbered affiliations see end of article.

Correspondence to

Dr Lan Wang;

lanwang@tongji.edu.cn

\section{ABSTRACT}

Objectives The aim was to elucidate the relationship between liver function and idiopathic pulmonary arterial hypertension (IPAH).

Design and setting Retrospective, longitudinal study in urban tertiary care centre in Shanghai, China.

Participants 407 IPAH consecutive incident patients age 18-65 years were retrospectively enrolled from January 2008 to December 2018.

Outcome measurements The primary endpoint was all-cause mortality. The cut-off value was determined by receiver operating characteristic curve (ROC), which was validated by Cox proportional hazard model was internally validated by bootstrap analysis and used for survival analysis. The Cox model was (internally) validated and cross-validated areas under the curve (AUC) should be reported.

Results The prevalence of abnormal liver function tests (LFTs) at baseline was $77.6 \%$. Hyperbilirubinaemia is the most common abnormal biochemical liver test: abnormal total bilirubin (TBIL in $51.6 \%$ patients). During the follow-up, 160 patients died. Patients with mixed liver dysfunction have worse prognosis than those with normal LFTs or isolated abnormal bilirubin metabolism. Comparing with patients with hepatocellular injury, the survival of patients with abnormal bilirubin metabolism is lower. Multivariable Cox models revealed a positive association between TBIL, $\gamma$-glutamyltransferase (GGT) and mortality showing that each Ig increment in TBIL and GGT was associated with a higher all-cause mortality (TBIL: HR 4. 29 (95\% Cl 1. 21 to 15. 27), p=0. 02; GGT: HR 2. 76 (95\% Cl 1.18 to 6. 45), $p=0.02)$. A novel formula named Liver Function Predict Index (LFPI) was constructed (LFPI $\left.=-0.002 * 6 \mathrm{MWD}+1.014^{*} \lg \mathrm{GGT}+1.458^{*} \mathrm{lg} \mathrm{TBIL}\right)$ to predict prognosis. ROC curve analysis did further identify 2.729 as the best cut-off value for LFPI (AUC 0.75, $p<0.001$, sensitivity $79 \%$, specificity $70 \%$ ).

Conclusions Liver dysfunction is frequent in IPAH, and characterised by a predominantly cholestatic enzyme profile. LFTs abnormalities are associated with worse survival and LFPI was a new and simple predictor for prognosis of IPAH.

\section{Strengths and limitations of this study}

- It is a retrospective cohort study in China to elucidate the relationship between liver function and idiopathic pulmonary arterial hypertension.

- A novel formula was conducted for predicting prognosis in idiopathic pulmonary arterial hypertension according to the multivariate cox proportional hazard model.

- All data came from a single centre indicated the possibility of selection and statistical bias.

\section{INTRODUCTION}

Idiopathic pulmonary arterial hypertension (IPAH) is a severe disease characterised by progressive loss and remodelling of the pulmonary arteries resulting in right heart failure and death. ${ }^{1}$ Without treatment, the median survival is less than 3 years. ${ }^{2}$ In the past few years, treatment of IPAH has undergone an extraordinary evolution and the survival has improved. ${ }^{3-5}$ However, IPAH remains a progressive and fatal disease, especially to patients with WHO functional class (WHO FC) III or IV, the risk of severe right heart failure or sudden cardiac death increased significantly compared with those of class I or II. ${ }^{36}$ This may be at least partly attributable to interactions between IPAH and other organs. $^{7}$

Liver function abnormalities are common in heart failure and are related to a poor outcome. $^{8-10}$ Passive congestion and impaired perfusion of liver, which is believed to be causative mechanisms of "cardiohepatic syndromes', ${ }^{11}$ could be probably found in IPAH. ${ }^{12}$ So far, the effects of bilirubin, alanine aminotransferase (ALT) and aspartate aminotransferase (AST) on IPAH are 
elucidated in a few studies. These studies suggest hyperbilirubinaemia could predict severity and outcomes of IPAH patients. ${ }^{713}$ However, many indicators of liver function were not included.

Thus, we performed this study to identify the characteristic pattern of liver function test (LFT) abnormalities in a large well-defined cohort of consecutive patients with IPAH and to comprehensively and systematically evaluate the relationships between LFTs, severity and survival in IPAH.

\section{METHODS}

\section{Study patients}

Four hundred and seven consecutive patients, aged 18-65 years old, newly diagnosed with IPAH in Department of Cardio-Pulmonary Circulation, Shanghai Pulmonary Hospital from January 2008 to December 2018 were retrospectively evaluated. To assess survival, patients were observed by the study investigators in the outpatient clinic or by telephone interview until 30 December 2019 (time of data censoring) or occurrence of death. During the follow-up, $25(6.1 \%)$ patients were lost. Censoring proportion are $60.7 \%$.

Right heart catheterisation (RHC) was performed for confirming PAH: (1) mean pulmonary arterial pressure (m PAP) $\geq 25 \mathrm{~mm} \mathrm{Hg}$; (2) pulmonary artery wedge pressure (PAWP) $\leq 15 \mathrm{~mm} \mathrm{Hg}$; (3) pulmonary vascular resistance (PVR) $>3$ wood units; (4) ruling out other causes of $\mathrm{PAH}$, the diagnosis of IPAH was established by at least two experienced PAH experts according to 2015 European Society of Cardiology (ESC)/ European Respiratory Society (ERS) pulmonary hypertension guidelines. ${ }^{14-16}$ The exclusion criteria were as follows: (1) patients with other types of pulmonary hypertension; (2) a history of hepatobiliary diseases, chronic nephritis, chronic renal dysfunction or active malignancy; (3) alcohol abuse and (4) possible hepatotoxic medication or drug-induced liver dysfunction. Epidemiological, demographics, clinical data, treatment, RHC and LFT were extracted from medical records using a standardised data collection form. All data were checked by two physicians independently and a third researcher adjudicated any difference in interpretation between the two primary reviewers.

\section{Six minute walk distance and RHC}

The 6 min walk distance (6MWD) test was performed according to the recommendations of the American Thoracic Society (2002).${ }^{17}$ Haemodynamic evaluation by RHC was performed at baseline in all subjects according to the previously described protocol. ${ }^{16} 18$ The mean right atrial pressure (m RAP), m PAP, PAWP, PVR and cardiac output $(\mathrm{CO})$ calculated by thermodilution were established. The cardiac index (CI) was calculated as the ratio of $\mathrm{CO}$ to the body surface area.

\section{Liver function tests}

All LFTs were measured at baseline by immune projection turbidimetry on ADVIA 2400 clinical chemistry system (Siemens Healthcare Diagnostic, German), at the biochemistry laboratory of Shanghai Pulmonary Hospital. Parameters of liver function include three aspects: ALT (upper limit of normal (ULN) 49U/L), AST (ULN $34 \mathrm{U} / \mathrm{L}$ ), lactate dehydrogenase (LDH, ULN 246U/L) and alkaline phosphatase (AKP, ULN 129U/L) are indicators of hepatocellular injury; serum total bilirubin (TBIL, ULN 17. $1 \mu \mathrm{mol} / \mathrm{L}$ ), direct bilirubin (DBIL, ULN $6 \mu \mathrm{mol} / \mathrm{L}$ ), indirect bilirubin (IDBIL, ULN 10. $2 \mu \mathrm{mol} / \mathrm{L}$ ) and $\gamma$-glutamyltransferase (GGT, ULN $38 \mathrm{U} / \mathrm{L}$ ) are indicators of bilirubin metabolism; Albumin (ALB, lower limit of normal (LLN) $34 \mathrm{~g} / \mathrm{L}$ ) and albumin-bilirubin (ALBI, calculated as $\lg$ TBIL $[\mu \mathrm{mol} / \mathrm{L}] \times 0.66-\mathrm{ALB}[\mathrm{g} / \mathrm{L}] \times 0$. $085)$ are indicators of liver reserve function. ALBI, grade $1 / 2 / 3$ are named as follows: the value $\leq-2.60$ (grade 1 ), more than -2.6 to $\leq-1.39$ (grade 2 ) and the value more than -1.39 (grade 3 ). ${ }^{19}$ The higher the grade, the worse the liver reserve function. ${ }^{19}{ }^{20} \mathrm{Abnormal} \mathrm{LFTs} \mathrm{were}$ defined as values above the ULN or below the LLN. In addition, ratios as follows were calculated. ALT /AKP $<2$ suggests biliary tract obstruction, $>5$ for hepatocellular injury and $2 \leq$ the ratio $\leq 5$ hints both. ${ }^{21}$ GGT /AST $>3$ also suggests biliary tract obstruction. ${ }^{21}$ Different types of jaundice were expressed by ratio of DBIL/TBIL: the value $<0$. 2 (haemolytic jaundice), the value $>0.5$ (obstructive jaundice), more than 0.2 to $\leq 0.5$ (hepatocellular jaundice). ${ }^{22}$

\section{Statistical analysis}

Baseline variables were described using mean $\pm S D$, medians and IQR for continuous variables and percentages for categorical variables. Shapiro-Wilk test was used to test the normality of continuous variables. Comparisons of means between two independent groups were performed by unpaired Student's t-test and Mann-Whitney U test for normally and not normally distributed samples, respectively. The categorical variables were expressed as frequency and percentage and were compared using Pearson's $\chi^{2}$ or Fisher's exact test depending on the expected counts in the table cells. As the data of LFTs were normally distributed, so we used log-transformed data for the following statistical analysis. The correlation between LFTs and clinical variables were assessed using partial correlation coefficients, adjusted for age, gender and body mass index (BMI). Univariate and multivariate COX proportional hazards models were examined to evaluate the HRs for all-cause mortality with 95\% CI. Sex-stratified multivariable Cox proportional hazards models were constructed. We included all covariates that correlated with the outcome using stepwise selection, with thresholds of $\mathrm{p}<0.05$ for entry and 0.10 for removal from the model. C-index was performed according to Harrell for assessment of the predictive accuracies of the multivariable Cox regression models. The proportional hazards assumption by testing statistical significance of interactions between follow-up time and variables for potential 
time-variant biases, which showed that none were significant based on a $p$ value threshold of 0.05 . C-index was performed according to Harrell for assessment of the predictive accuracies of the multivariable Cox regression models and the value is 0.72 . The internal validity of the model was assessed by bootstrapping. ${ }^{23}$ In 1000 bootstrap samples, the expected shrinkage of the final regression model was calculated. ${ }^{24}$ The fivefold cross-validation was used to identify Liver Function Predict index (LFPI) for predicting the survival of IPAH patients, and the average areas under the curve (AUC) value was calculated. The DeLong method ${ }^{25}$ was used for comparison of AUCs. Receiver operating characteristic (ROC) curves were constructed to explore the optimum cut-off value that maximised sensitivity and specificity. Survival curves were derived by the Kaplan-Meier method and compared by the log-rank test. For all analyses, a $p$ value $<0.05$ was considered as statistically significant. The main analysis was performed by SPSS IBM) V.22.0 and Python V.3.8.

Patients and public involvement

No patient involved.

\section{RESULTS}

A total of 170 of the 577 patients were excluded (online supplemental figure 1). Of the 170 patients, 108 patients were excluded from the study because of the age (53 were aged under 18 years old and 55 were aged above 65 years old), the other 62 patients were excluded followed the exclusion criteria (20 had a history of hepatobiliary diseases, 13 possible have drug-induced liver dysfunction, 6 with alcohol abuse, 5 have chronic hepatitis and 8 were chronic nephritis). Hence, 407 IPAH patients were included in the study. Baseline characteristics of study patients were shown in table 1 and figure 1A-C. Four hundred and seven patients were included with a mean age of $37 \pm 13$ years old. Seventy-one per cent patients $(\mathrm{n}=289)$ were female and $66.6 \%$ patients were in WHO FC III/IV. Most of patients (92.1\%) received PAH-targeted medications, and $39.8 \%$ received combination therapy.

\section{Baseline LFT levels in IPAH}

Three hundred and sixteen patients $(77.6 \%)$ had abnormal LFTs, 34 patients with isolated bilirubin metabolism dysfunction, 13 with isolated hepatocellular injury

\begin{tabular}{|c|c|c|c|c|}
\hline Variables & All $(n=407)$ & Survivor $(n=247)$ & Non-survivor $(n=160)$ & P value* \\
\hline Age, years & $37 \pm 13$ & $38 \pm 12$ & $38 \pm 13$ & 0.88 \\
\hline Female & 289(71) & $189(76.5)$ & $100(62.5)$ & 0.005 \\
\hline $\mathrm{BMI}, \mathrm{kg} / \mathrm{m}^{2}$ & $22.19 \pm 3.20$ & $22.12 \pm 3.19$ & $22.25 \pm 3.26$ & 0.72 \\
\hline 6MWD, m & $380 \pm 111$ & $405.14 \pm 104.55$ & $341.04 \pm 109.68$ & $<0.001$ \\
\hline WHO FC & & & & 0.002 \\
\hline Class I/II & $136(33.4)$ & $95(38.5)$ & $41(25.6)$ & \\
\hline Class III/IV & 271 (66.6) & 154 (62.3) & 117 (73.1) & \\
\hline Targeted drugs & 375 (92.1) & $234(94.7)$ & $141(88.1)$ & 0.02 \\
\hline PDE-5 inhibitors & $151(37.1)$ & $94(38.1)$ & $57(35.6)$ & 0.62 \\
\hline ERAs & $44(10.7)$ & $23(9.3)$ & $21(13.1)$ & 0.23 \\
\hline Prostacyclin analogue & $10(2.5)$ & $5(2)$ & $5(3.1)$ & 0.48 \\
\hline Soluble guanylate cyclase stimulator & $8(2)$ & $5(2)$ & $3(1.9)$ & 0.92 \\
\hline Combination therapy, n (\%) & $162(39.8)$ & $107(43.3)$ & $55(34.3)$ & 0.07 \\
\hline \multicolumn{5}{|l|}{ Haemodynamic assessments } \\
\hline m RAP, $\mathrm{mm} \mathrm{Hg}$ & $7.21 \pm 5.21$ & $6.2 \pm 4.4$ & $9.0 \pm 5.9$ & $<0.001$ \\
\hline m PAP, mm Hg & $59.3 \pm 14.3$ & $56.6 \pm 12.7$ & $63.4 \pm 15.6$ & $<0.001$ \\
\hline PVR, wood units & $14.1 \pm 6.4$ & $12.7 \pm 5.5$ & $16.5 \pm 6.9$ & $<0.001$ \\
\hline $\mathrm{CO}, \mathrm{l} / \mathrm{min}$ & $4.1 \pm 1.4$ & $4.4 \pm 1.4$ & $3.7 \pm 1.2$ & $<0.001$ \\
\hline $\mathrm{Cl}, \mathrm{l} / \mathrm{min} / \mathrm{m}^{2}$ & $2.6 \pm 0.8$ & $2.7 \pm 0.9$ & $2.2 \pm 0.6$ & $<0.001$ \\
\hline
\end{tabular}

Continuous variables were using means $( \pm S D)$ and medians $(I Q R)$ for normally and not normally distributed samples, respectively. $n(\%)$ is described for categorical variables.

Italic values were used when $P$ values $<0.05$.

${ }^{*}$ The differences in continuous variables between survival and dead patients were determined by Student's t-test and Mann-Whitney $U$ test for normally and not normally distributed samples, respectively. The differences in categorical variables were compared using Pearson's $\chi 2$ or Fisher's exact test depending on the expected counts in the table cells.

ERAs, endothelin receptor antagonists; $\mathrm{m} \mathrm{BMI}$, body mass index; $\mathrm{Cl}$, cardiac index; $\mathrm{CO}$, cardiac output; WHO FC, WHO function classification; IPAH, idiopathic pulmonary arterial hypertension; m PAP, mean pulmonary artery pressure; 6MWD, six min walk test distance; PDE-5, phosphodiesterase type 5; PVR, pulmonary vascular resistance; m RAP, mean right atrial pressure. 

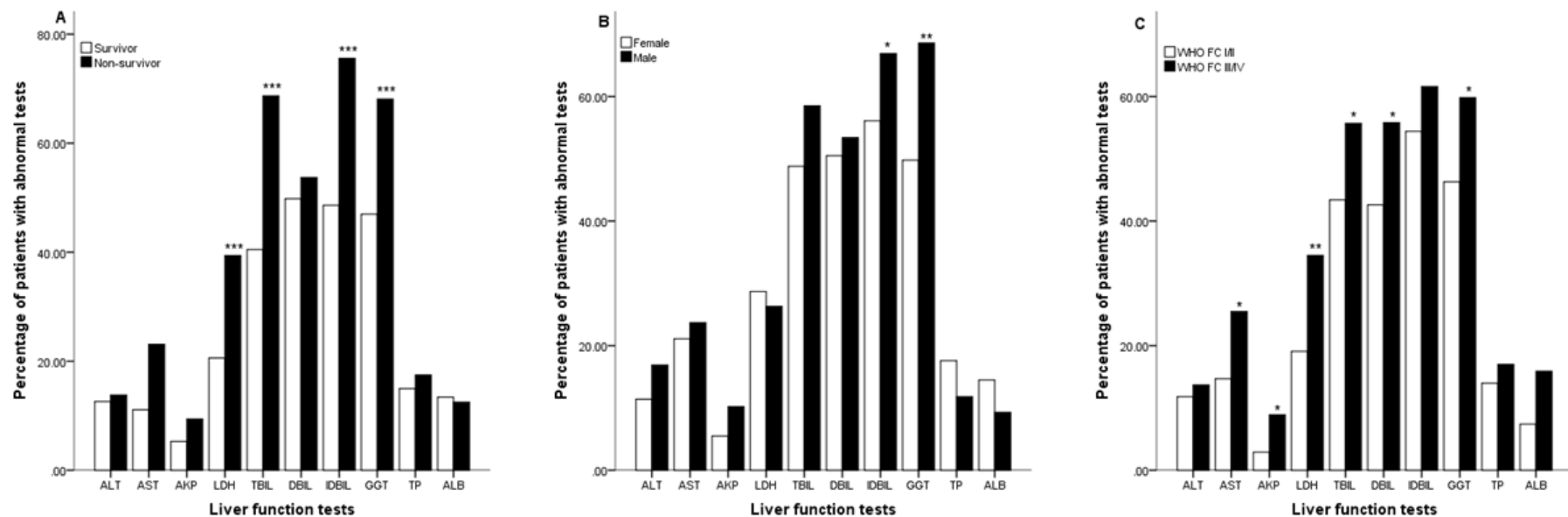

Figure 1 Prevalence of baseline live function test abnormalities in idiopathic pulmonary arterial hypertension patients. ALB, albumin; AKP, alkaline phosphatase; ALT, alanine aminotransferase; AST, aspartate aminotransferase; DBIL, direct bilirubin; IDBIL, indirect bilirubin; GGT, $\gamma$-glutamyltransferase; LDH, lactate dehydrogenase; TBIL, total bilirubin; TP, total protein; WHO $\mathrm{FC}, \mathrm{WHO}$ functional class.

and 8 with isolated synthetic dysfunction (reduced ALB), 261 with mixed liver dysfunction. The most common LFT abnormalities were hyperbilirubinaemia (67\%), including $51.6 \%$ patients with higher TBIL, 51.4\% patients with higher DBIL and $59.2 \%$ patients with higher IDBIL, followed by elevated GGT $55.3 \%$, elevated LDH $28 \%$, reduced albumin $13 \%$. Abnormal AST and ALT were observed in $11.9 \%$ and $13 \%$ IPAH patients, respectively. Only $6.9 \%$ patients have elevated AKP (table 2).

Compared with the female patients, the prevalence of abnormal IDBIL (66. $9 \%$ vs $56.1 \%$; $\mathrm{p}=0.04$ ) and GGT $(68.6 \%$ vs $49.8 \%$; $\mathrm{p}<0.001)$ was larger in male patients (figure 1B and online supplemental table 1). Patients with WHO FC III/IV had higher proportion of elevated TBIL $(55.7 \%$ vs $43.4 \%$; p=0.02), GGT $(59.8 \%$ vs $46.3 \%$; $\mathrm{p}=0.01)$, LDH ( $34.5 \%$ vs $19.1 \%$; $\mathrm{p}=0.005)$, and decreased ALB (15.9\% vs 7.4\%; $\mathrm{p}=0.02)$ than those with WHO FC I/ II (figure 1C and online supplemental table 1).

\section{Association between LFTs and severity of IPAH}

Partial correlation analysis showed that LFTs abnormalities were associated with the severity of IPAH. In general, the higher WHO FC, m RAP and PVR, the lower the 6MWD and CO, the more abnormal for the LFTs, but the correlation is weak. Correlations between bilirubin (DBIL and TBIL), GGT and $\mathrm{m}$ RAP ( $\mathrm{r}=0.507,0.480$ and 0.369 separately; all $\mathrm{p}<0.001$,) were stronger than the other LFT parameters. Among them, serum TBIL and DBIL were correlated with only one haemodynamic index (m RAP) while GGT was correlated with three haemodynamic indexes (m RAP, PVR and CO) in IPAH (all $\mathrm{p}<0.01$, table 3). There was no association between $\mathrm{m}$ PAP and LFTs (all $p>0.05$ ) (table 3 ). However, in female patients, higher $\mathrm{m}$ PAP was accompanied with higher TBIL, DBIL and IDBIL $(\mathrm{r}=0.624, \mathrm{p}<0.001 ; \mathrm{r}=0.492, \mathrm{p}=0.005 ; \mathrm{r}=0.548$, $\mathrm{p}=0.001$ separately) (online supplemental table 2). Yet, in male patients, there was no correlation between $\mathrm{m}$ PAP and bilirubin (TBIL, DBIL and IDBIL) (online supplemental table 3).
Relationships between LFTs at baseline and survival in IPAH The mean duration of follow-up was $51 \pm 34$ months (median 48 months, IQR 20-78 months). A total of $160(39.3 \%)$ patients died of right heart failure and the 1-year, 3-year, 5-year and 10-year survival rates were $89.4 \%$, $78.1 \%, 68.3 \%, 62.2 \%$ and $60.9 \%$, respectively. Abnormality of baseline LFTs is more common in non-survival patients (table 2 and figure 1A).

Patients with GGT/AST $>3$ had lower survival rate than patients with GGT/AST $\leq 3(\mathrm{p}<0.001$ by log-rank analysis) (figure 2A). Patients with DBIL/TBIL $>0.5$ had worse prognosis than DBIL/TBIL $<0.2$ ( $\mathrm{p}=0.03$ by log-rank analysis) (figure 2B). There was no association between ALT/ AKP and ALBI, grade and survival (figure 2C,D). Patient with isolated reduced ALB and mixed liver dysfunction had higher mortality than those with normal liver function $(\mathrm{p}=0.01$ and $\mathrm{p}<0.001$, respectively, figure $2 \mathrm{E}$ ). Patients with mixed liver dysfunction have worse prognosis than those with normal LFTs or isolated abnormal bilirubin metabolism, meanwhile patients with isolated albumin deficiency also have higher mortality than those with normal LFTs (figure 2E). There was no difference in survival rate among patients with isolated reduced ALB, isolated biliary metabolism anomaly and isolated hepatocellular injury (figure 2E).

Univariate cox proportional hazard analysis showed higher bilirubin (HR and 95\% CI: 4.74 (2.68 to 8.38) for per $\lg$ TBIL; 3.15 (2.07 to 4.82) for per lg DBIL and 3.92 (2.12 to 7.27) for per lg IDBIL), GGT4.50 (2.93 to 6.91) for per lg GGT) and ratio of GGT/AST (1.15 (1.11 to 1.2)) increased risk of death in IPAH $(\mathrm{p}<0.001)$ (table 4$)$. When referring to hepatocellular injury parameters, elevated AST (HR 2.43 for per lg AST; p=0.03), LDH (HR 12.14 for per $\lg \mathrm{LDH} ; \mathrm{p}<0.001$ ) and AKP (HR 6.47 for per lg AKP; $\mathrm{p}<0.001$ ) were also predictors of worse prognosis. However, no association was found between the indicators (ALT/AKP, ALB and ALBI, grade) and survival (table 4 and figure 2D). In multivariable Cox model, 
Table 2 Characteristics of liver function tests at baseline in IPAH patients

\begin{tabular}{|c|c|c|c|c|}
\hline Variables* & All $(n=407)$ & Survivor $(n=247)$ & Non-survivor $(n=160)$ & P value \\
\hline ALT, U/L & 25 (17-35) & $24(17-34)$ & $26(18-37)$ & 0.26 \\
\hline Abnormal (>ULN) & $53(13)$ & $31(12.6)$ & $22(13.8)$ & 0.73 \\
\hline Abnormal (>ULN) & 89 (11.9) & $52(11.1)$ & $37(23.1)$ & 0.62 \\
\hline AKP, U/L & $73(57-96)$ & 68 (54.8-86.5) & $83(61-104)$ & $<0.001$ \\
\hline LDH, U/L & 218 (182-264) & 207 (173.5-244.3) & 234 (195-277) & $<0.001$ \\
\hline Abnormal (>ULN) & $114(28)$ & $51(20.6)$ & $63(39.4)$ & $<0.001$ \\
\hline TBIL, $\mu \mathrm{mol} / \mathrm{L}$ & $19(12-27)$ & $16(11-22)$ & $23(16-34.8)$ & $<0.001$ \\
\hline Abnormal (>ULN) & $210(51.6)$ & $100(40.5)$ & $110(68.7)$ & $<0.001$ \\
\hline DBIL, $\mu \mathrm{mol} / \mathrm{L}$ & $6(4-10)$ & $5(3.4-7.6)$ & $8.6(4-13.9)$ & $<0.001$ \\
\hline Abnormal (>ULN) & 241 (59.2) & $120(48.6)$ & $121(75.6)$ & $<0.001$ \\
\hline GGT, U/L & $43(25-79)$ & $34(20-60.8)$ & 64 (34-109) & $<0.001$ \\
\hline Abnormal (>ULN) & $225(55.3)$ & $116(47)$ & $109(68.1)$ & $<0.001$ \\
\hline TP, g/L & $65(60-70)$ & $65(60-69)$ & $65(59-70)$ & 0.90 \\
\hline Abnormal (<LLN) & $65(16)$ & $37(15)$ & $28(17.5)$ & 0.50 \\
\hline ALB, $g / L$ & $39(36-42)$ & $39(36-42)$ & $39.5(37-42)$ & 0.10 \\
\hline Abnormal (<LLN) & $53(13)$ & $33(13.4)$ & $20(12.5)$ & 0.80 \\
\hline ALBI, Grade & & & & 0.29 \\
\hline Grade1 & $170(41.8)$ & 105 (42.5) & $65(40.6)$ & \\
\hline GGT/AST>3 & $102(25.1)$ & $43(17.4)$ & 59 (36.9) & $<0.001$ \\
\hline DBIL/TBIL & & & & 0.07 \\
\hline DBIL/TBIL <0. 2 & $39(9.6)$ & $23(9.3)$ & $16(10)$ & \\
\hline $0.2 \leq \mathrm{DBIL} / \mathrm{TBIL} \leq 0.5$ & $333(81.8)$ & $209(84.6)$ & $124(77.5)$ & \\
\hline DBIL/TBIL>0. 5 & $35(8.6)$ & $15(6)$ & $20(12.5)$ & \\
\hline
\end{tabular}

Values are means $( \pm \mathrm{SD})$ and $\mathrm{n}(\%)$ for continuous variables and categorical variables, respectively.

Italic values were used when $P$ values $<0.05$.

*Liver function tests abnormal defined as lower/higher than lower/upper limits of normal. The normal ranges are 10-49 U/L for ALT, 0-34 U/L

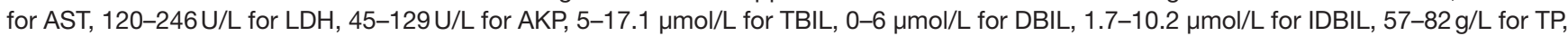
34-50 g/L for ALB.

†The differences in continuous variables between survival and dead patients were determined by Mann-Whitney $U$ test. The differences in categorical variables were compared using Pearson's $\chi 2$ or Fisher's exact test depending on the expected counts in the table cells. AKP, alkaline phosphatase; ALB, albumin; ALT, alanine aminotransferase; AST, aspartate aminotransferase; DBIL, direct bilirubin; GGT, $\gamma$-glutamyl transferase; ALBI, Grade, albumin-bilirubin, Grade; IDBIL, indirect bilirubin; IPAH, idiopathic pulmonary arterial hypertension; LDH, lactate dehydrogenase; TBIL, total bilirubin; TP, total protein.

independently of established clinical markers including age, BMI, sex, WHO FC class, treatment and haemodynamics, TBIL and GGT retained their prognostic power, and were inversely associated with survival (4. 29 (1.21 to 15. 27) for per lg TBIL, GGT 2. 756 (1. 177 to 6.451$)$ for per $\lg$ GGT; both $\mathrm{p}=0$. 02) (table 4). The internal validity of the model was assessed by bootstrapping. ${ }^{23}$ After bootstrapped shrinkage, all variables identified by Cox regression were retained and the expected shrinkage is 0.96 . 
Table 3 Correlations between liver function test at baseline and haemodynamic parameters, WHO FC and 6 MWD*

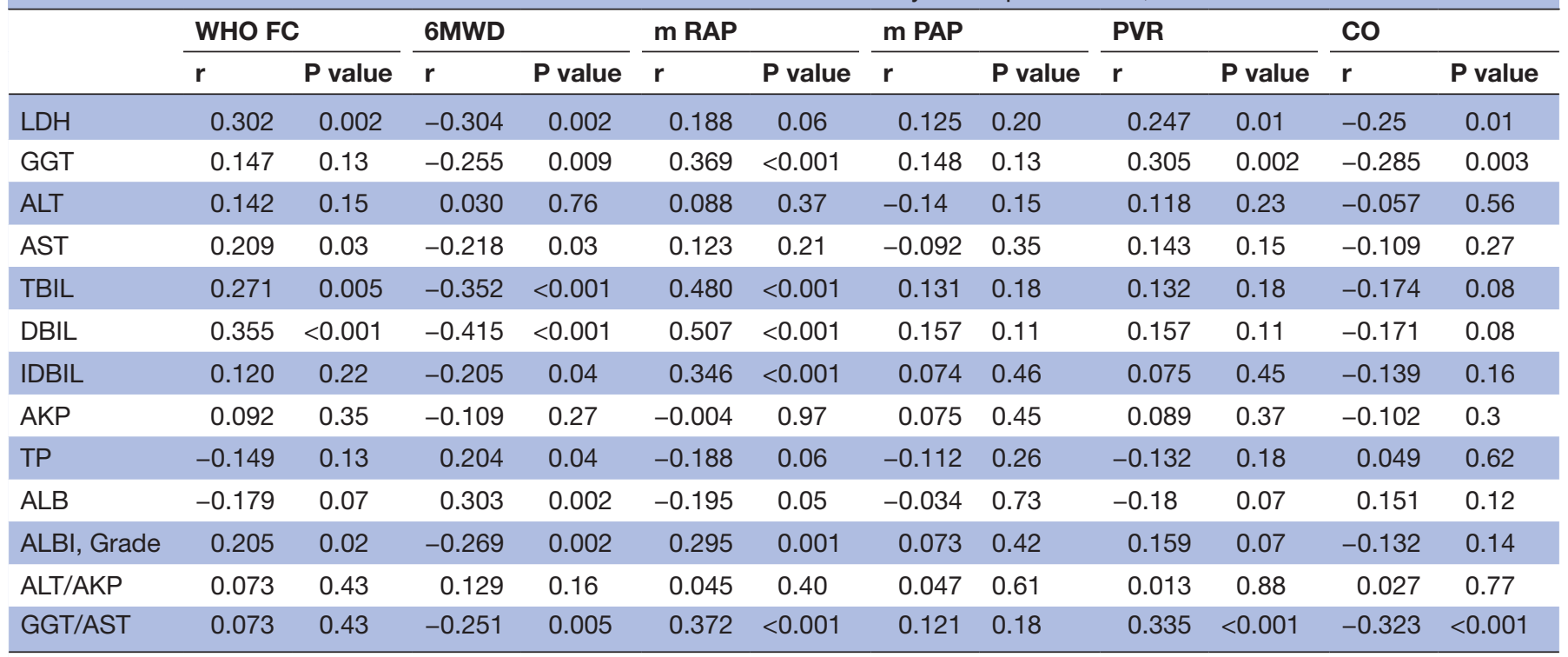

${ }^{*}$ Association between liver function test (LFT) and clinical variables were calculated by partial correlation coefficients with recursive method, adjusted for age, gender and BMI. Log-transformed value of LFTs was used in these statistical analysis.

AKP, alkaline phosphatase; ALB, albumin; ALT, alanine aminotransferase; AST, aspartate aminotransferase; BMI, body mass index; CO, cardiac output; DBIL, direct bilirubin; WHO FC, WHO function classification; GGT, $\gamma$-glutamyl transferase; ALBI, Grade, albumin-bilirubin, Grade; IDBIL, indirect bilirubin; LDH, lactate dehydrogenase; 6MWD, six min walk test distance; m PAP, mean pulmonary artery pressure; PVR, pulmonary vascular resistance; $m$ RAP, mean right atrial pressure; TBIL, total bilirubin; TP, total protein.

The average AUC value after fivefold cross-validation was 0.75 .

ROC curve analysis further identified $18.8 \mu \mathrm{mol} / \mathrm{L}$ as the optimal cut-off level for TBIL (area under the ROC curve (AUC) $0.68 ; \mathrm{p}<0.001$; sensitivity $69 \%(95 \%$ CI $61 \%$ to $76 \%$ ); specificity $62 \%$ (95\% CI $56 \%$ to $68 \%$ ) (figure 3). Patient with higher baseline TBIL ( $>18.8$ $\mu \mathrm{mol} / \mathrm{L})$ had significantly worse survival $(\mathrm{p}<0.001$ by logrank analysis; figure $4 \mathrm{~A}$ ), so did the patients with higher GGT $(>57.5 \mathrm{IU} / \mathrm{L}$ (the best cutoff value according to ROC curve analysis), AUC 0.69, $\mathrm{p}<0.001$; sensitivity $55 \%$ (95\% CI $46 \%$ to $62 \%$ ); specificity $74 \%$ (95\% CI $68 \%$ to $79 \%) ; p<0.001$ by log-rank analysis) (figures 3 and $4 \mathrm{~B}$ ). In order to increase the sensitivity and specificity of LFTs for predicting survival of IPAH, we herein constructed a new formula named with LFPI to predict prognosis, according to the multivariate cox proportional hazard model: $\quad$ LFPI $=-0.002 * 6 \mathrm{MWD}+1.014 * \mathrm{lg} \quad$ GGT $+1.458 * \lg$ TBIL, where 6MWD was given in metre, GGT in $\mathrm{U} / \mathrm{L}$ and TBIL in $\mu \mathrm{mol} / \mathrm{L}$.ROC curve analysis did further identify 2.729 as the best cut-off value for LFPI (AUC 0.75, $\mathrm{p}<0.001$, sensitivity $79 \%$ (95\% CI $72 \%$ to $85 \%$ ); specificity $70 \%$ (95\% CI $64 \%$ to $76 \%$ ). Figure 3 also shows that LFPI was numerically superior to 6MWD (AUC 0.67; $95 \% \mathrm{CI} 0.62$ to 0.73 ). One-hundred and twenty-seven IPAH subjects with higher LFPI died, compared with 33 of those with LFPI $<2.729$. Patients with LFPI $<2.729$ had better prognosis (log-rank test $\mathrm{p}<0.001$; figure $4 \mathrm{C}$ ). The estimated 1-year, 3-year, 5-year and 10-year survival was $94.2 \%, 90.1 \%, 84.3 \%$ and $80.8 \%$, respectively, in patients with LFPI $<2.729$. It was $85.6 \%, 69.1 \%$ and $56.4 \%$ and $46.2 \%$, respectively, in patients with LFPI $\geq 2.729$.

\section{DISCUSSION}

For the first time, our study evaluated three aspects of liver function (including hepatocellular injury, bilirubin metabolism and liver reserve function) and the relationship between LFTs and severity, and prognosis in IPAH patients. This analysis identified that (1) abnormality of baseline LFTs is common in IPAH, mainly characterised by hyperbilirubinaemia; (2) patients with mixed liver dysfunction, isolated albumin deficiency or abnormal bilirubin metabolism have worse prognosis; (3) TBIL and GGT were independently associated with mortality of IPAH and (4) A new index was conducted, named as LFPI $(=-0.002 * 6 \mathrm{MWD}+1.014 * \lg$ GGT $+1.458 * \lg$ TBIL $)$, which is numerically superior to 6MWD for predicting prognosis.

To date, the prognostic significance of liver function abnormalities in heart failure has been well recognised, ${ }^{8-10}$ but there are limited studies to investigate the association between LFT and IPAH. A retrospective study suggested elevated serum bilirubin was a risk factor for death in patients with pulmonary arterial hypertension. ${ }^{7}$ However, this small study only included 37 patients and did not systematic describe the characterisation and significance of liver function abnormalities in IPAH. Another study with 404 IPAH patients demonstrated that serum DBIL could predict severity and outcome in IPAH. ${ }^{13}$ However, this study only comprised four indicators (ALT, AST, 

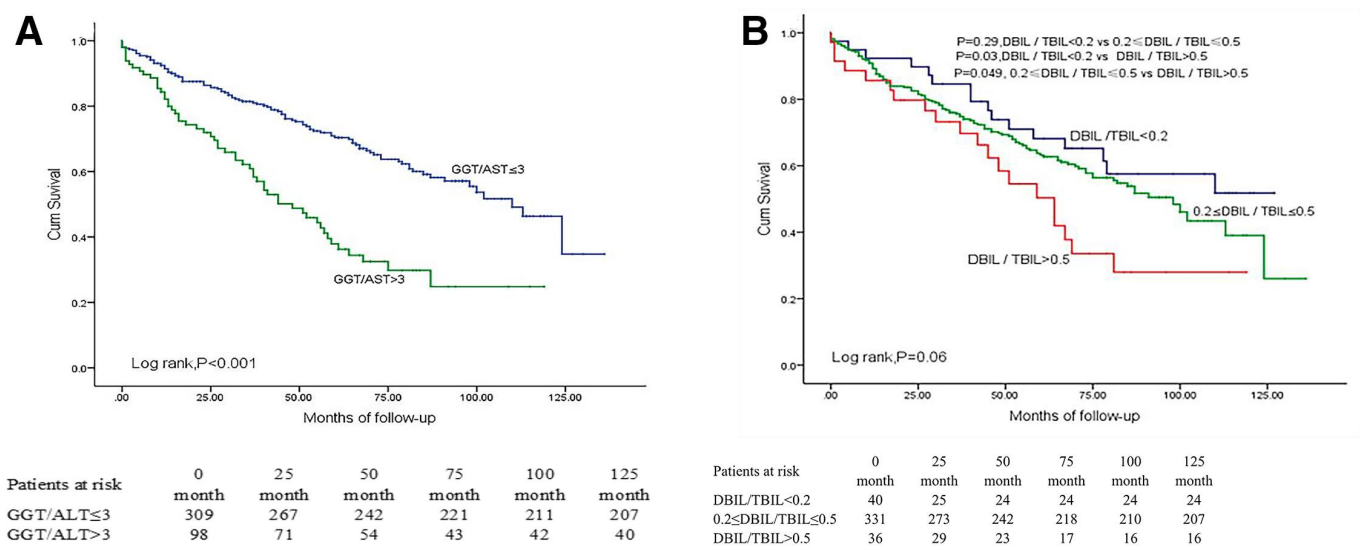

\section{C}
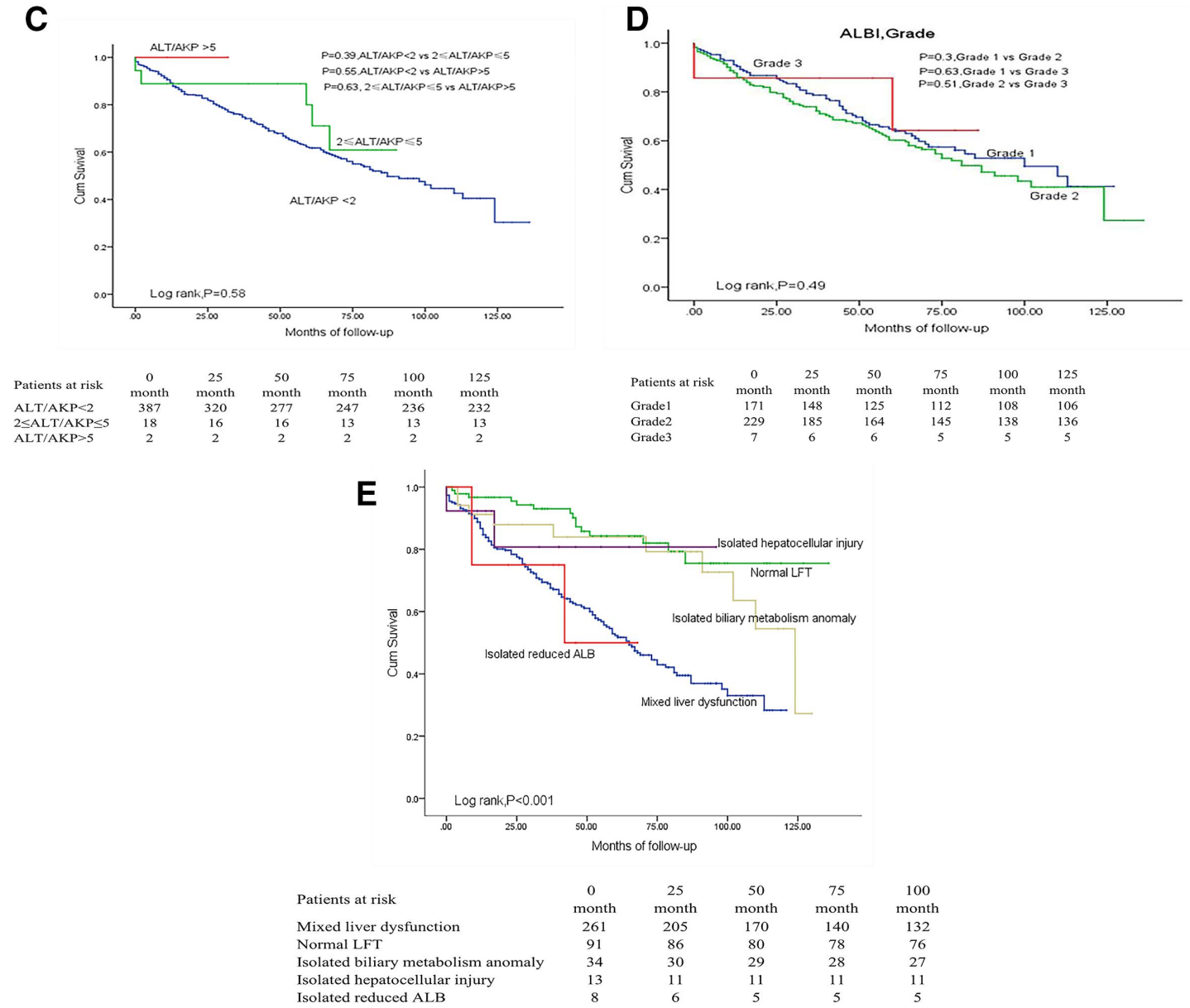

Figure 2 Influence of levels of liver function ratio and type of liver function abnormality on clinical outcome of patients with idiopathic pulmonary arterial hypertension patients. AKP, alkaline phosphatase; ALB, albumin; ALBI, albumin-bilirubin; ALT, alanine aminotransferase; DBIL, direct bilirubin; GGT, $\gamma$-glutamyltransferase; LFT, liver function test; TBIL, total bilirubin.

TBIL and DBIL) and did not comprehensively elucidate the characterisation of liver function abnormalities in IPAH. These above studies just focused on aspect of bilirubin metabolism. Our study provided a comprehensive evaluation of the prevalence and prognostic importance of LFT abnormalities in IPAH, and the analysis comprised three aspects: hepatocyte injury, dysfunction of bilirubin metabolism and liver reserve function. Apart from that, some ratios were used in our study to elucidate liver function in sideways. We found that hyperbilirubinaemia was positively associated with severity and inversely associated with prognosis in IPAH, which is similar with other IPAH studies. In addition, we also researched some other aspects of LFT, and findings were as follows: first, the prevalence of abnormal LFTs at baseline in IPAH patients was $77.6 \%$. Hyperbilirubinaemia occurs in $67 \%$ patients and is the most common abnormal biochemical liver test in patients with IPAH. The percentage of abnormal GGT $(55.3 \%)$, as well as ALT, and AST AKP, ALB in IPAH was similar with those in left heart failure. ${ }^{86-33}$ Much higher 
Table 4 Univariate and multivariate Cox regression analysis with overall survival

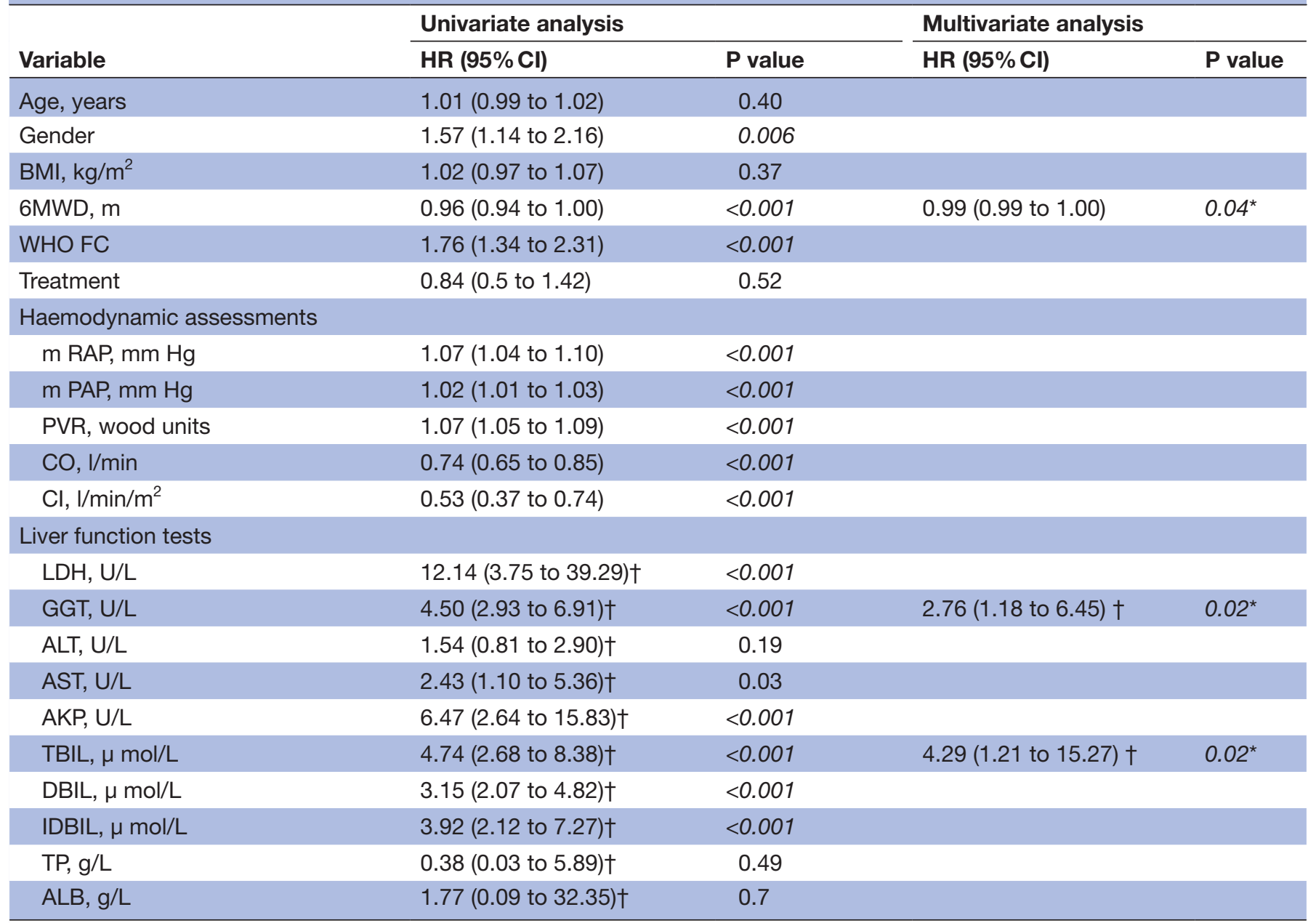

Italic values were used when $P$ values $<0.05$.

${ }^{*} \mathrm{P}$ values represent the results of Cox proportional regression analysis adjusted for age, gender, treatment and BMI. †HR for every 1 -lg increase in LFTs.

ALBI, albumin-bilirubin; AKP, alkaline phosphatase; ALB, albumin; ALT, alanine aminotransferase; AST, aspartate aminotransferase; BMI, body mass index; Cl, cardiac index; CO, cardiac output; DBIL, direct bilirubin; WHO FC, WHO function classification; GGT, $\gamma$-glutamyl transferase; IDBIL, indirect bilirubin; LDH, lactate dehydrogenase; LFT, liver function test; 6MWD, six min walk test distance; m PAP, mean pulmonary artery pressure; PVR, pulmonary vascular resistance; $m$ RAP, mean right atrial pressure; TBIL, total bilirubin; TP, total protein.

percentage of abnormal TBIL (51.6\%) in IPAH patients was observed in our data compared with TBIL in symptomatic chronic $(13 \%)$ and acute heart failure $(19 \%-$ $26 \%) .{ }^{28} 2933{ }^{34}$ Second, the higher WHO FC, m RAP and PVR, the lower the 6MWD and CO, the more abnormal for the LFTs, among them serum TBIL and DBIL were correlated with only one haemodynamic index (m RAP) while GGT was correlated with three haemodynamic indices (m RAP, PVR and CO) in IPAH. Third, some sex difference was found: the male patients had larger percentage of elevated IDBIL and GGT than the female ones with IPAH; m PAP was positively associated with hyperbilirubinaemia in female patients, while the correlation was not found in male patients. Fourthly, LFTs (except for ALT and albumin) and ratio of GGT/AST can predict survival. Furthermore, TBIL and GGT independently of established clinical markers including age, BMI, sex, WHO FC, treatment and haemodynamics retained their prognostic power in multivariable Cox regression, which is similar in $\mathrm{HF}^{32}{ }^{34-36}$ The last, according to multivariable Cox regression, we constructed a new formula named LFPI $(=-0.002 * 6 \mathrm{MWD}+1.014 * \lg \quad$ GGT $+1.458 * \lg$ TBIL $)$ to predict prognosis, which was numerically superior to 6MWD.

The mechanisms that cause serum TBIL and GGT elevated in IPAH remains uncertain. In our analysis, liver function abnormality is related to the severity of IPAH as assessed by WHO FC, 6MWD and haemodynamics, which is similar to the relationship between LFTs and heart failure. ${ }^{28} 2937{ }^{38} \mathrm{We}$ found serum TBIL, DBIL and GGT were well positively correlated with (m RAP) in IPAH. Giallourakis $e t a l^{39}$ described that hepatopathy secondary to chronic congestive HF is attributed to three main processes: increased hepatic venous pressure, decreased hepatic blood flow originating from low $\mathrm{CO}$ and decreased arterial oxygen saturation. ${ }^{40}$ In severe 


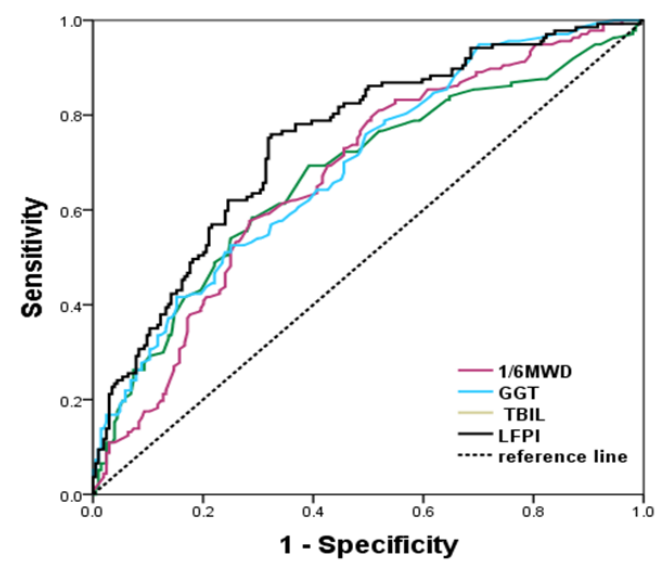

\begin{tabular}{|c|c|c|c|c|c|c|c|c|c|}
\hline & \multirow{2}{*}{ AUC } & \multicolumn{2}{|c|}{ TBIL } & \multicolumn{2}{|c|}{ GGT } & \multicolumn{2}{|c|}{ 1/6MWD } & \multicolumn{2}{|c|}{ LFPI } \\
\hline & & $z$ & $P$ & $z$ & $\mathrm{P}$ & $z$ & $P$ & $z$ & $P$ \\
\hline TBIL & 0.67 & - & - & 0.48 & 0.63 & 0.02 & 0.98 & 1.83 & 0.06 \\
\hline GGT & 0.69 & 0.48 & 0.63 & - & - & 0.46 & 0.64 & 1.36 & 0.17 \\
\hline 1/6MWD & 0.67 & 0.02 & 0.98 & 0.46 & 0.64 & - & - & 1.84 & 0.07 \\
\hline LFPI & 0.75 & 1.83 & 0.06 & 1.36 & 0.17 & 1.84 & 0.07 & - & - \\
\hline
\end{tabular}

Figure 3 Receiver operating characteristic curves showing the ability of total bilirubin (TBIL), $\gamma$-glutamyltransferase (GGT), 6 min walk distance (6MWD) and Liver Function Predict Index (LFPI) to predict survival in idiopathic pulmonary arterial hypertension patients. AUC, areas under the curve.

IPAH patients, which may be similar to chronic congestive HF, we proposed that elevated right heart filling pressure (including right atrial pressure)and central venous pressures (CVPs) are transmitted through the hepatic veins and into the small hepatic venules. ${ }^{40}$ The effect of this transmitted pressure is passive congestion of the liver resulting in elevated hepatic venous pressure, which can impair delivery of oxygen and nutrients to hepatocytes, leading to hepatocyte necrosis with subsequent atrophy of liver cells and oedema of the peripheral area. ${ }^{27324041}$ Thus, patients with the more severe passive congestion and the greater reduction in forward flow were more likely to have an abnormality in LFTs. Previous studies have shown that changes to bilirubin during heart failure maybe caused by haemodynamic alterations, which is related to both CVP and CI. ${ }^{72627}$ Poelzl et $a l^{4}$ have demonstrated that TBIL and GGT independently correlated with tricuspid regurgitation. Elevated CVP, RAP and the severity of tricuspid regurgitation could cause high hepatic venous pressure, ${ }^{28}{ }^{40}$ then cause venous congestion and lead to cholestatic and hepatic liver injury. ${ }^{26} 2840$ Both cholestatic and hepatic hypoxic injury may contribute to elevated bilirubin and GGT in IPAH.

Our study showed that, there were only weak relationships between LFTs and haemodynamics, which suggests maybe there are other factors contributing to the extent of liver injury, such as the renin angiotensin system, the sympathetic nervous system as confirmed in $\mathrm{HF}$.

The prognostic significance of liver function abnormalities in heart failure has long been recognised, but the mechanisms linking LFT and poor outcomes in IPAH are still unclear. This study demonstrates that in patients with IPAH, all LFTs except for ALB and ALT predicted overall survival. Of these variables, TBIL and GGT retained their prognostic power after adjustment with multivariable Cox regression. Our results are consistent with previous studies that have focused on the relationship between LFT and heart failure. It is noteworthy that heart failure could lead to centrilobular hepatic necrosis, even cirrhosis, ${ }^{30} 323541$ whether hepatic cirrhosis could have further effect on the primary disease and if these constitute a vicious cycle. Cirrhosis often results in portal hypertension, and the hyperdynamic status may increase shear stress at the level of the pulmonary vasculature and the portosystemic shunts may exacerbate the imbalance between vasoconstrictor and vasodilator. ${ }^{39}$ However, long-term and larger sample studies are needed to confirm the interaction and mechanism between liver function and IPAH.
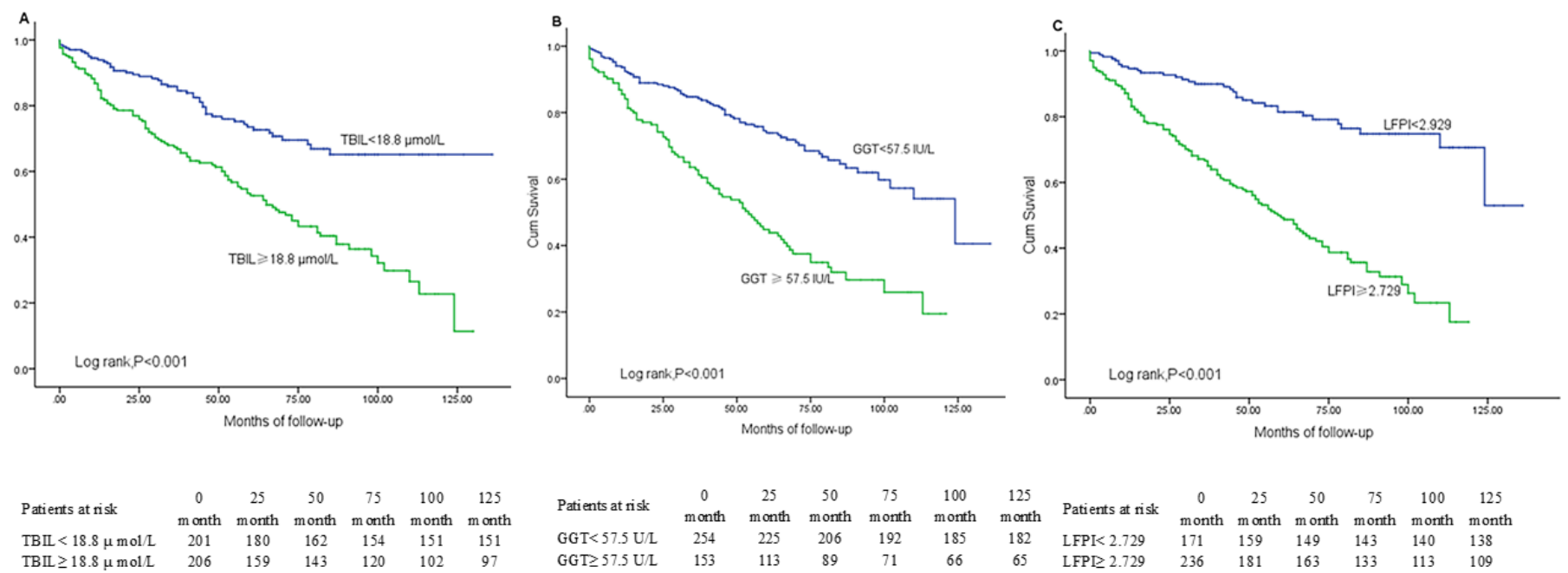

125

$\begin{array}{ccccccc}\text { Patients at risk } & \begin{array}{c}0 \\ \text { month }\end{array} & 25 & 50 & 75 & 100 & 125 \\ \text { month } & \begin{array}{c}\text { month } \\ \text { month }\end{array} & \text { month } & \text { month }\end{array}$

TBIL $\geq 18.8 \mu \mathrm{moll} \quad 206 \quad 159 \quad 143 \quad 120 \quad 102 \quad 97$

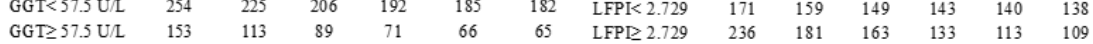

Figure 4 Kaplan-Meier curves and survival analysis in idiopathic pulmonary arterial hypertension patients according to total bilirubin (TBIL), $\gamma$-glutamyltransferase (GGT) and Liver Function Predict Index (LFPI). 


\section{Limitations}

Our study has several limitations, including its retrospective design and all data came from a single centre, which indicated the possibility of selection and statistical bias. Meanwhile, some unrealistically large HR estimates and confidence limits in our results suggest the possibility of sparse-data bias. ${ }^{42}$ Furthermore, dynamic changes in LFT were not included and we did not assess whether the observed abnormalities were transient or permanent. Although we found sex difference between hyperbilirubinaemia and IPAH, further researches are needed to confirm the result and elucidate the mechanism. We did not measure surrogates of neurohumoral or inflammatory activation, which are most likely to influence the mutual interaction between IPAH and liver. In addition, Prothrombin Time (PT) and International Normalized Ratio (INR) which representing reserve function of liver were not included in our analysis.

In conclusion, the prevalence of abnormal baseline LFTs was observed in $77.6 \%$ IPAH patients, characterised by impaired of bilirubin metabolism. Elevated TBIL and GGT are positively associated with severity and are independent predictors for poor prognosis in IPAH. A new formula named as LFPI $(=-0.002 * 6 \mathrm{MWD}+1.014 * \mathrm{lg}$ GGT $+1.458 * \lg$ TBIL) would be a simple index in terms of predicting survival. Therefore, LFTs, as a simple and widely used test would be useful to identify severity and prognosis in IPAH patients. However, long-term and larger sample studies are needed to confirm the outcomes and to elucidate the mechanism and interaction between liver function and IPAH.

\section{Author affiliations \\ ${ }^{1}$ Cardio-Pulmonary Circulation, Tongji University Affiliated Shanghai Pulmonary Hospital, Shanghai, China \\ ${ }^{2}$ Respiratory and Critical Care Medicine, Tongji University Affiliated Shanghai \\ Pulmonary Hospital, Shanghai, China \\ ${ }^{3}$ Shanghai Pulmonary Hospital, Tongji University Affiliated Shanghai Pulmonary Hospital, Shanghai, China}

Acknowledgements The authors gratefully acknowledge the contribution of all investigators who participated in this study. We also thank the patients who participated in the study.

Contributors CL, WW, CW and $\mathrm{HQ}$ contributed to the study design and operation, data acquisition, analysis and manuscript preparation. The four authors contributed equally and are the cofirst authors. PY, RJ, QZ, SG and RZ contributed to manuscript editing and revision. $\mathrm{JL}$ and $\mathrm{JH}$ contributed to study operation and data acquisition. $\mathrm{JL}$ and LW contributed to the study conception and design, revising the article critically for important intellectual content and final approval of the version to be published.

Funding This work was supported by the Programme of Shanghai Municipal Commission of Health (20204Y0384), Programme of Natural Science Foundation of Shanghai (18ZR1431500), Clinical Research Foundation of Shanghai Pulmonary Hospital (FKLY20005 and FKLY20011), Programme of the National Science and Technology Information System of the People's Republic of China (2018YFC1313603).

Competing interests None declared.

Patient consent for publication Not required.

Ethics approval The study protocol was approved by Shanghai Pulmonary Hospital ethics committee, and all participants provided written informed consent.
Provenance and peer review Not commissioned; externally peer reviewed.

Data availability statement Data are available on reasonable request.

Supplemental material This content has been supplied by the author(s). It has not been vetted by BMJ Publishing Group Limited (BMJ) and may not have been peer-reviewed. Any opinions or recommendations discussed are solely those of the author(s) and are not endorsed by BMJ. BMJ disclaims all liability and responsibility arising from any reliance placed on the content. Where the content includes any translated material, BMJ does not warrant the accuracy and reliability of the translations (including but not limited to local regulations, clinical guidelines, terminology, drug names and drug dosages), and is not responsible for any error and/or omissions arising from translation and adaptation or otherwise.

Open access This is an open access article distributed in accordance with the Creative Commons Attribution Non Commercial (CC BY-NC 4.0) license, which permits others to distribute, remix, adapt, build upon this work non-commercially, and license their derivative works on different terms, provided the original work is properly cited, appropriate credit is given, any changes made indicated, and the use is non-commercial. See: http://creativecommons.org/licenses/by-nc/4.0/.

\section{ORCID iDs}

Cijun Luo http://orcid.org/0000-0002-0239-5494

Rong Jiang http://orcid.org/0000-0003-4062-5550

\section{REFERENCES}

1 Farber HW, Loscalzo J. Pulmonary arterial hypertension. N Engl J Med Overseas Ed 2004;351:1655-65.

2 Jing Z-C, Xu X-Q, Han Z-Y, et al. Registry and survival study in Chinese patients with idiopathic and familial pulmonary arterial hypertension. Chest 2007;132:373-9.

3 Zhang R, Dai L-Z, Xie W-P, et al. Survival of Chinese patients with pulmonary arterial hypertension in the modern treatment era. Chest 2011;140:301-9.

4 Benza RL, Miller DP, Gomberg-Maitland M. Predicting survival in pulmonary arterial hypertension: insights from the registry to evaluate early and long-term pulmonary arterial hypertension disease management (reveal). Circulation 2010;122:164-72.

5 Humbert M, Sitbon O, Chaouat A, et al. Survival in patients with idiopathic, familial, and anorexigen-associated pulmonary arterial hypertension in the modern management era. Circulation 2010;122:156-63.

6 Barst RJ, McGoon M, Torbicki A, et al. Diagnosis and differential assessment of pulmonary arterial hypertension. J Am Coll Cardiol 2004;43:S40-7.

7 Takeda Y, Takeda Y, Tomimoto S, et al. Bilirubin as a prognostic marker in patients with pulmonary arterial hypertension. BMC Pulm Med 2010;10:22.

8 Klaudia V, Lenka S, Jindrich S. Prevalence and clinical significance of liver function abnormalities in patients with acute heart failure. Biomed Papers 2015;159:429-36.

9 Andrew PA, Timothy PD, Paul AH. Effect of minor liver function test abnormalities and values within the normal range on survival in heart failure. Am J Cardio 2015;115:938-41.

10 Jan B, Hans LH, Douwe P. Abnormal liver function tests in acute heart failure: relationship with clinical characteristics and outcome in the protect study. Euro J Heart Fail 2016;18:830-9.

11 Naschitz JE, Slobodin G, Lewis RJ, et al. Heart diseases affecting the liver and liver diseases affecting the heart. Am Heart $J$ 2000:140:111-20.

12 Megalla S, Holtzman D, Aronow WS, et al. Predictors of cardiac hepatopathy in patients with right heart failure. Med Sci Monit 2011;17:CR537-41.

13 XQ X, ZC L, Liu QQ. Direct bilirubin: a new risk factor of adverse outcome in idiopathic pulmonary arterial hypertension. Inter $\mathrm{J}$ Cardio 2017;228:895-9.

14 Galie N, Humbert M, Vachiery JL. ESC/ERS guidelines for the diagnosis and treatment of pulmonary hypertension: the joint Task force for the diagnosis and treatment of pulmonary hypertension of the European Society of cardiology (ESC) and the European respiratory Society (ERS) endorsed by: association for European paediatric and congenital cardiology (AEPC), International Society for heart and lung transplantation (ISHLT). Eur Respir $J$ 2015;2015:903-75.

15 Simonneau G, Robbins IM, Beghetti M, et al. Updated clinical classification of pulmonary hypertension. J Am Coll Cardiol 2009;54:S43-54. 
16 Simonneau G, Gatzoulis MA, Adatia I, et al. Updated clinical classification of pulmonary hypertension. J Am Coll Cardiol 2013;62:D34-41

17 statement ATS. Guidelines for the six-minute walk test. Am J Respir Crit Care Med 2002;166:111-7.

18 Sitbon O, Humbert M, Jaïs X, et al. Long-term response to calcium channel blockers in idiopathic pulmonary arterial hypertension. Circulation 2005;111:3105-11.

19 Johnson PJ, Berhane S, Kagebayashi C, et al. Assessment of liver function in patients with hepatocellular carcinoma: a new evidencebased approach-the ALBI grade. JCO 2015;33:550-8.

20 Ho S-Y, Liu P-H, Hsu C-Y, et al. Comparison of twelve liver functional reserve models for outcome prediction in patients with hepatocellular carcinoma undergoing surgical resection. Sci Rep 2018;8:4773.

21 LI F, LU L. Evaluation of abnormal liver function and its clinical significance. J Clin Hepatol 2015;31:1543-6.

22 YQ O. Clinical diagnostics. Beijing: People's medical publishing house, 2005: 52

23 Brookman-May S, May M, Shariat SF, et al. Features associated with recurrence beyond 5 years after nephrectomy and nephron-sparing surgery for renal cell carcinoma: development and internal validation of a risk model (PRELANE score) to predict late recurrence based on a large multicenter database (CORONA/SATURN project). Eur Urol 2013;64:472-7.

24 Harrell FE, Lee KL, Mark DB. Multivariable prognostic models: issues in developing models, evaluating assumptions and adequacy, and measuring and reducing errors. Stat Med 1996;15:361-87.

25 DeLong ER, DeLong DM, Clarke-Pearson DL. Comparing the areas under two or more correlated receiver operating characteristic curves: a nonparametric approach. Biometrics 1988;44:837-45.

26 van Deursen VM, Damman K, Hillege HL, et al. Abnormal liver function in relation to hemodynamic profile in heart failure patients. $J$ Card Fail 2010;16:84-90.

27 Lau GT, Tan HC, Kritharides L. Type of liver dysfunction in heart failure and its relation to the severity of tricuspid regurgitation. Am J Cardiol 2002;90:1405-9.

28 van Deursen VM, Edwards C, Cotter G, et al. Liver function, inhospital, and post-discharge clinical outcome in patients with acute heart failure-results from the relaxin for the treatment of patients with acute heart failure study. J Card Fail 2014;20:407-13.
29 Ambrosy AP, Vaduganathan M, Huffman MD, et al. Clinical course and predictive value of liver function tests in patients hospitalized for worsening heart failure with reduced ejection fraction: an analysis of the Everest trial. Eur J Heart Fail 2012;14:302-11.

30 Auer J. What does the liver tell us about the failing heart? Eur Heart $J$ 2013;34:711-4.

31 Maria N, Parissis J, Birhan YM. Liver function abnormalities, clinica profile, and outcome in acute decompensated heart failure. Eur Heart J 2013:34:742-9.

32 Samsky MD, Patel CB, DeWald TA, et al. Cardiohepatic interactions in heart failure. J Am Coll Cardiol 2013;61:2397-405

33 Allen LA, Felker GM, Pocock S, et al. Liver function abnormalities and outcome in patients with chronic heart failure: data from the candesartan in heart failure: assessment of reduction in mortality and morbidity (CHARM) program. Eur J Heart Fail 2009;11:170-7.

34 Poelzl G, Ess M, Mussner-Seeber C, et al. Liver dysfunction in chronic heart failure: prevalence, characteristics and prognostic significance. Eur J Clin Invest 2012;42:153-63.

35 Poelzl G, Eberl C, Achrainer H, et al. Prevalence and prognostic significance of elevated $\gamma$-Glutamyltransferase in chronic heart failure. Circulation 2009;2:294-302.

36 Ruttmann E, Brant LJ, Concin H. Gamma-Glutamyl transferase as a risk factor for cardiovascular disease mortality: an epidemiological investigation in a cohort of 163,944 Austrian adults. Circulation 2005;112:2130-7.

37 Targher G, Byrne CD. Circulating markers of liver function and cardiovascular disease risk. Arterioscler Thromb Vasc Biol 2015;35:2290-6.

38 Dunn GD, Hayes P, Breen KJ, et al. The liver in congestive heart failure: a review. Am J Med Sci 1973;265:174-90.

39 Giallourakis CC, Rosenberg PM, Friedman LS. The liver in heart failure. Clin Liver Dis 2002;6:947-67.

40 Giusca S, Jinga M, Jurcut C, et al. Portopulmonary hypertension: from diagnosis to treatment. Eur J Intern Med 2011;22:441-7.

41 Alvarez A, Mukherjee D, Debabrata M. Liver abnormalities in cardiac diseases and heart failure. International Journal of Angiology 2011;20:135-42.

42 Greenland S, Mansournia MA, Altman DG. Sparse data bias: a problem hiding in plain sight. BMJ 2016;352:i1981. 